\title{
Analysis of Pedestrian Accidents in Irbid City, Jordan
}

\author{
Bashar H. Al-Omari*, ${ }^{* 1}$ and Eman S. Obaidat ${ }^{2}$ \\ ${ }^{I}$ Jordan University of Science \& Technology, P.O. Box (3030), Irbid, Jordan \\ ${ }^{2}$ Ministry of Public Works and Housing, Amman, Jordan
}

\begin{abstract}
The numbers of people who are walking and bicycling continues to increase all over the world and pedestrian accidents are becoming a serious problem in the developed and developing countries. However, the problem is more severe in the developing countries, such as Jordan where pedestrians represented $32.5 \%$ of all traffic accident fatalities and $23.8 \%$ of all traffic accident injuries during the year 2010. This study aims at analyzing pedestrian accidents in Irbid city, as an example for urban areas in Jordan, based on a total of 1090 pedestrian accidents that have occurred in the City during the three years period (1999 - 2001). The analysis was based on pedestrian characteristics, driver characteristics, accident time, accident location, weather, road surface condition, illumination condition, vehicle characteristics, speed limit, accident severity, pedestrian faults, and driver faults. Geographic Information System (GIS) tool was used to locate all pedestrian accidents over the study area. The results of the study showed that the majority of pedestrian accidents have occurred at non-intersection locations, during clear and sunny weather, on dry surface, during daylight, and at low speed limits. Also, more pedestrian accidents have occurred during the afternoons, on Thursdays, and during July. The majority of involved drivers in pedestrian accidents were males, with private license type, driving private vehicles and committed the fault of "not giving priority to pedestrians". The majority of pedestrian victims were males, children less than 15 years old, and hit by vehicles while crossing the road.
\end{abstract}

Keywords: Pedestrian, accidents, driver, vehicle, speed.

\section{INRODUCTION}

Statistics reported by the Jordan Traffic Institute [1] indicated that 140,014 traffic accidents have occurred during the year 2010, which have resulted into a total of 670 deaths and 17,403 injuries. Pedestrians were involved during the same period in 4091 traffic accidents that have resulted into 218 fatalities and 4140 injuries. It was noticed that the majority of pedestrian accidents in Jordan have occurred in urban areas.

Irbid city was selected as an example for urban areas in Jordan to investigate the characteristics of pedestrian accidents based on pedestrian characteristics, driver characteristics, accident time, accident location, weather, road surface condition, illumination condition, vehicle characteristics, speed limit, accident severity, pedestrian faults, and driver faults. Irbid City has the second largest metropolitan population in Jordan after the capital city, Amman, with a population of around 300,000 (becomes 650,000 if the Greater city area is considered), and is located about $70 \mathrm{~km}$ to the north of Amman. The city is a major ground transportation hub between Jordan and Syria in the north. Irbid region is also home to several colleges and universities, the two most prominent being Jordan University of Science and Technology and Yarmouk University. The study area used in the investigation was the main city area as shown in Fig. (1).

*Address correspondence to this author at the Jordan University of Science \& Technology, P.O. Box (3030), Irbid, Jordan; Tel: +962-2-7201000 (22102); Fax:+962 - 2 - 7095123; E-mail: bahomari@just.edu.jo
This research aims at analyzing pedestrian accidents in Irbid city based on pedestrian characteristics (age and gender), driver characteristics (age, gender, and license type), accident time (hour, day, and month), accident location (midblock, intersection \& bus terminal), weather, road surface condition, illumination condition, vehicle characteristics, speed limit, accident severity, pedestrian faults, and driver faults.

The characteristics of pedestrian accidents were investigated for the three years period (1999-2001). Data on traffic accidents were obtained from traffic directorate, public security headquarters, Jordan. Each pedestrian accident was located on Irbid streets map, using geographic information system (GIS) software to relate the accidents with their location characteristics.

\section{LITRATURE REVIEW}

The number of people who are walking and bicycling continues to increase all over the world and are currently forming around 12 percent of trips in the United States [2]. As a result, pedestrian accidents are becoming a serious problem in the developed and developing countries.

Pedestrians represented $32.5 \%$ of all traffic accident fatalities and $23.8 \%$ of all traffic accident injuries in Jordan [1] during the year 2010. The problem is worse in some other developing countries like Egypt where around $75 \%$ of the traffic injuries are related to accidents involving pedestrians [3].

These are considered high figures as compared to the developed countries. Based on (2007-2009) statistics, 


\section{ANALYSIS OF PEDESTRIAN ACCIDENTS IN IRBID CITY, JORDAN}

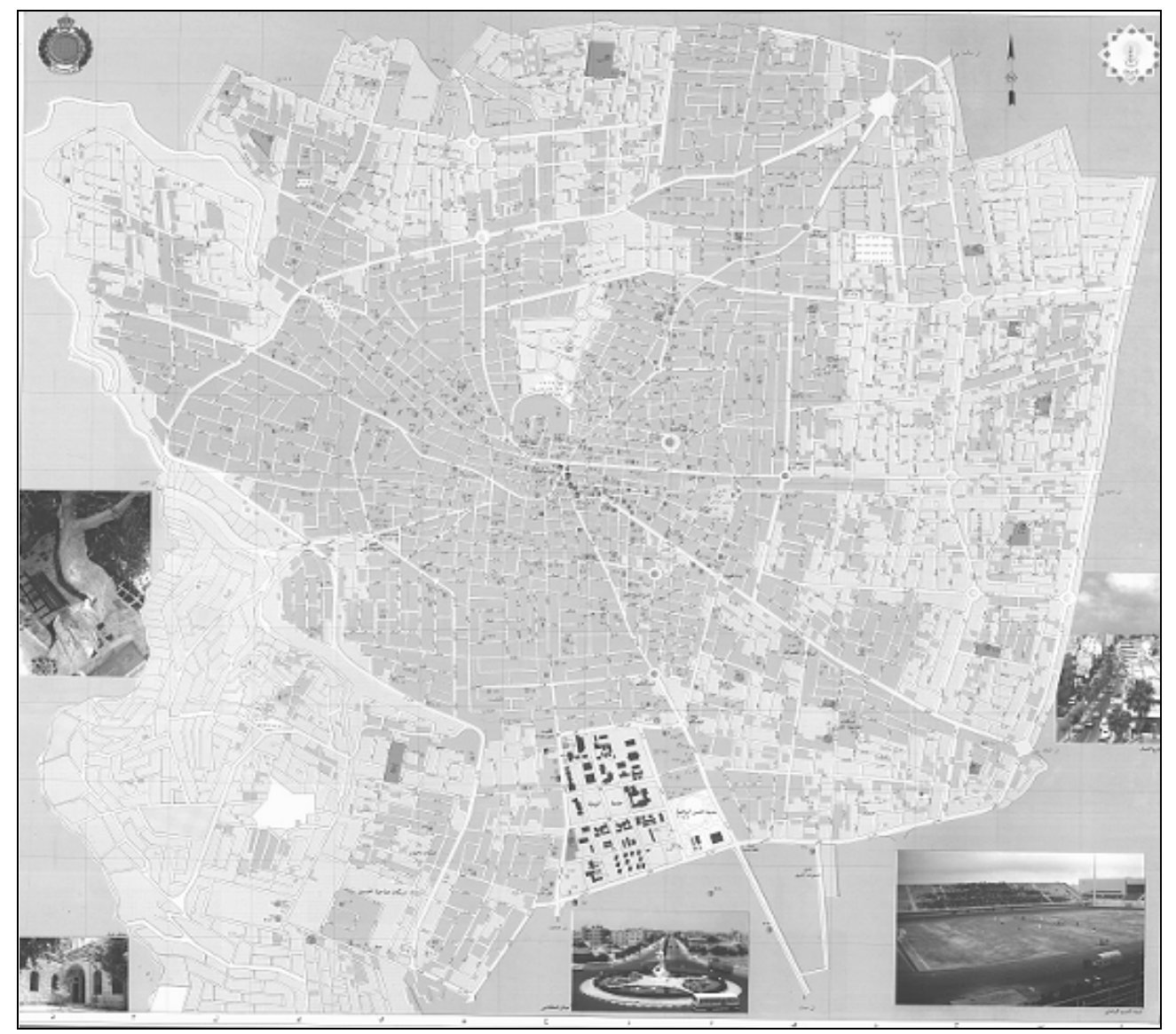

Fig. (1). Irbid City study area (Source: Irbid Municipality).

pedestrians represented $20 \%$ of all road deaths across the EU countries ranging from around $9 \%$ in the Netherlands to $37 \%$ in Romania [4]. In USA [5], a total of 4,280 pedestrians were killed (13\% of all traffic fatalities) and 70,000 were injured (3\% of all traffic injuries) during the year 2010. This could be explained by the fact that, in the developed countries, almost every pedestrian is a driver that makes him/her more familiar with the traffic environment [6]. On the other hand, in most of the developing countries, there is a low auto ownership, so more journeys are made on foot resulting in more likelihood of people being on the streets facing the risk of being hit by vehicles.

WHO states that around $90 \%$ of the global road traffic mortality occurs in low- and middle-income countries [7]. Zegeer and Bushell [8] indicated that pedestrians are more susceptible to traffic crashes in countries where traffic laws are poorly enforced and that pedestrians are most at risk in urban areas due to the large amount of vehicle and pedestrian activities. Wickramasinghe et al. [9] have noticed that in developing countries, even when there are sufficient pedestrian road crossing facilities, pedestrians take the risk and follow illegal road crossing patterns increasing their exposure to traffic accidents.

Several studies investigated the characteristics of pedestrian accidents. For example, LaPlante et al. [10] investigated the characteristics of pedestrian accidents in Michigan and found that young pedestrians ages 5 to 24 are more likely to be involved in crashes, and that pedestrian crashes occur more often after dark than during daylight hours. Viola et al. [11] investigated the characteristics of crashes resulting in pedestrians killed or seriously injured in New York and found that nearly 36\% were due to driver inattention, $79 \%$ involved private automobiles, and $80 \%$ involved male drivers. Nieuwesteeg and McIntyre [12] found that injured pedestrians are usually not at fault when crossing at intersections, but mostly at fault when crossing the road mid-blocks in Victoria. Truong, and Somenahalli [13] have used the GIS tool for the analysis of pedestrian-vehicle crash data for identification and ranking of unsafe bus stops in Australia.

\section{DATA COLLECTION AND REDUCTION}

Data on pedestrian traffic accidents were obtained from traffic directorate, public security head quarters, Irbid. After reviewing the traffic accident reports for the three years period (1999-2001), a total of 1090 pedestrian accident reports were separated from around 10,000 traffic accident reports that included all types of traffic accidents [14]. The following are the major data items that existed in the traffic accident report and might be used in analyzing pedestrian traffic accidents: 
1. Location of the accident, based on a written description and a sketch.

2. Pedestrian age, gender and action (disregarding pedestrian traffic light, crossing from an incorrect location, crossing in front of a parked vehicle, working, playing, walking on the road, pushing a carriage, crossing at a zebra crossing, waiting to ride, getting in or out of a vehicle, crossing a pedestrian traffic light, getting in or out of a school bus, walking on sidewalks, riding a bicycle, off road, crossing the road, and walking inside a bus terminal).

3. Driver age, gender, license type, and fault (driving opposite to traffic direction, incorrect overtaking, exceeding speed limit, using incorrect lane, incorrect bending and turning, driving under the influence of alcohol, tail gating, incorrect parking, failing to comply with obligatory signs, disregarding a traffic light signal, not giving priority to vehicles, not giving priority to pedestrians, and incorrect reversing).

4. Vehicle category (motor cycle, agricultural vehicle, construction vehicle, small passenger car, minibus, bus, truck, dual-purpose vehicle, and special purpose vehicle) and vehicle type (foreign, government, private, public, and recreation).

5. Road surface condition (dry, wet, snowy, and icy), illumination condition (daylight, night with sufficient light, night with insufficient light, and dark) and weather condition (clear, fog, rain, snow, strong wind, and dust).

6. Accident severity (slight injury, medium injury, severe injury, and fatal).

7. Time of the day, day of the week, and month of the year.

\section{Speed limit.}

The Geographic Information System (GIS) has been used for the analysis and recording of pedestrian traffic accidents. Using GIS, the locations of traffic accidents were drawn as points on Irbid city map. After locating the accidents at the proper locations, each year had a separate layer (theme) over the base layer (the map). After that, all other characteristics of the accidents were added.

\section{ANALYSIS AND RESULTS}

To increase the efficiency of pedestrian's traffic safety improvement programs, the traffic safety officials need to focus on pedestrian and driver categories and consider the conditions that are directly related to pedestrian accidents. This requires more understanding of the relationship between pedestrian accidents and pedestrian characteristics, driver characteristics, accident time, accident location, weather, road surface condition, illumination condition, vehicle characteristics, speed limit, accident severity, pedestrian faults, and driver faults.

According to age groups. The highest relative involvement of the pedestrian casualties (by age groups and per 100,000 population within each age group) was for the age groups (6-10), (0-5), (11-15), \& (56-60) respectively as shown in Table 1 . Children below 15 years old who formed
$43 \%$ of the city population, accounted for around $64 \%$ of pedestrian accidents. Similar results were found for Jordan by Al-Masaeid [16].

Table 1. Pedestrian Causalities by Age Group and Per 100000 Population

\begin{tabular}{|c|c|c|c|}
\hline Age Group & Population & $\begin{array}{c}\text { Pedestrian } \\
\text { Causalities }\end{array}$ & $\begin{array}{c}\text { Relative Involvement } \\
\text { Per 100,000 Population }\end{array}$ \\
\hline \hline$\leq 5$ & 38528 & 269 & 698.19 \\
\hline $6-10$ & 35986 & 285 & 791.97 \\
\hline $11-15$ & 33251 & 172 & 517.28 \\
\hline $16-20$ & 30525 & 102 & 334.15 \\
\hline $21-25$ & 27696 & 73 & 263.58 \\
\hline $26-30$ & 21475 & 68 & 316.65 \\
\hline $31-35$ & 15091 & 41 & 271.69 \\
\hline $36-40$ & 10501 & 24 & 228.55 \\
\hline $41-45$ & 7870 & 11 & 139.77 \\
\hline $46-50$ & 6917 & 19 & 274.69 \\
\hline $51-55$ & 6401 & 14 & 218.72 \\
\hline $56-60$ & 5295 & 19 & 358.83 \\
\hline$>60$ & 41284 & 33 & 79.93 \\
\hline All & 280820 & 1130 & 402.39 \\
\hline
\end{tabular}

According to accident location. Accident locations were divided into intersections, mid blocks, and bus terminals. Intersection accident location was defined as the one that has occurred within $20 \mathrm{~m}$ of an intersection [15]. The majority of pedestrian accidents (69.4\%) have occurred at mid blocks, and a total of $26.8 \%$ have occurred at intersections. The rest of pedestrian accidents $3.8 \%$ have occurred inside bus terminals. Mid blocks are at more risk because of higher speeds, on street parking, and interruptions to both drivers and pedestrians. The unorganized traffic at terminals has caused a considerable number of pedestrian accidents.

According to road surface condition. Higher accident rates have occurred on dry surface conditions $(95.5 \%)$ as compared to wet surface conditions. This was expected because pedestrians tend to avoid walking on the streets under rainy conditions.

According to illumination condition. The majority of pedestrian accidents $(82.1 \%)$ have occurred during daylight condition, a bout $15.6 \%$ have occurred during nighttime with sufficient light, $2.0 \%$ have occurred during nighttime with insufficient light and only $0.3 \%$ have occurred during dark condition. These results are expected because this study was conducted for the urban area of the city where most of pedestrian trips end with the sunset. A study in Saudi Arabia presented similar results and showed that for most cities, the majority of accidents occurred during the day time [17].

According to accident severity. Pedestrian traffic accidents have resulted into $73.5 \%$ of slight injuries, $18.7 \%$ of medium injuries, $5.1 \%$ of severe injuries and $2.7 \%$ of fatalities. 
According to pedestrian gender. Males accounted for about $72.3 \%$ of pedestrian involvements in traffic accidents. This is due to higher mobility of the male population in the Middle Eastern countries. In Kuwait, more than $80 \%$ of pedestrian victims were males [18].

According to time of the day. It was found that accidents are more frequent during (12:00 - 3:00) PM with 28.3\% followed by $(3: 00-6: 00)$ PM with $23.9 \%$ as shown in Table 2. These time intervals represent the peak traffic and pedestrian activities in the City. It should be noted that the interval $(1: 00-2: 00)$ PM had the highest pedestrian accidents rate because it is the end of the working hours for most of public agencies and schools in Jordan.

Table 2. Pedestrian Accidents by Time of the Day

\begin{tabular}{|c|c|}
\hline Time of Day & \% of Pedestrian Accidents \\
\hline \hline $00: 00-03: 00$ & 1.28 \\
\hline $03: 00-06: 00$ & 0.27 \\
\hline $06: 00-09: 00$ & 8.72 \\
\hline $09: 00-12: 00$ & 14.59 \\
\hline $12: 00-15: 00$ & 28.26 \\
\hline $15: 00-18: 00$ & 23.85 \\
\hline $18: 00-21: 00$ & 15.87 \\
\hline $21: 00-24: 00$ & 7.16 \\
\hline Total & 100.00 \\
\hline
\end{tabular}

According to day of the week. It was found that pedestrian accidents are more frequent during Thursdays $16.5 \%$, and Sundays $16.1 \%$ as shown in Table 3. These two days represent the end and the start of the working days before and after the weekend in Jordan. These two days are characterized by more traffic and pedestrians activities.

Table 3. Pedestrian Accidents by Day of the Week

\begin{tabular}{|c|c|}
\hline Day & \% of Pedestrian Accidents \\
\hline \hline Saturday & 14.86 \\
\hline Sunday & 16.06 \\
\hline Monday & 14.50 \\
\hline Tuesday & 15.05 \\
\hline Wednesday & 13.21 \\
\hline Thursday & 16.51 \\
\hline Friday & 9.81 \\
\hline Total & 100.00 \\
\hline
\end{tabular}

According to month of the year. Monthly distribution of pedestrian accidents indicated that July is the peak month $(11.8 \%)$ as shown in Table 4. This agrees with Al-Momani [19] study for Amman City in Jordan. This summer month with long, warm, and active days, and with school holiday, in addition to the presence of large number of visiting
Jordanian expatriates, have more pedestrian and vehicle activities.

Table 4. Pedestrian Accidents by Month of the Year

\begin{tabular}{|c|c|}
\hline Month & \% of Pedestrian Accidents \\
\hline \hline January & 8.81 \\
\hline February & 7.71 \\
\hline March & 9.91 \\
\hline April & 6.88 \\
\hline May & 7.43 \\
\hline June & 8.53 \\
\hline July & 11.83 \\
\hline August & 8.81 \\
\hline September & 7.89 \\
\hline October & 5.78 \\
\hline November & 8.90 \\
\hline December & 7.52 \\
\hline Total & 100.00 \\
\hline
\end{tabular}

According to weather condition. The majority of pedestrian accidents (94.2\%) have occurred during clear weather condition and only $4.6 \%$ of pedestrian accidents have occurred during rainy conditions.

According to vehicle category. The majority of pedestrian accidents $(73.4 \%)$ are caused by small passenger cars, followed by dual-purpose vehicles $(12.0 \%)$ and then trucks $(7.8 \%)$ as shown in Table 5. This was expected because small passenger cars form the largest component of the traffic fleet in Jordan especially in urban areas.

Table 5. Pedestrian Accidents According to Vehicle Categories

\begin{tabular}{|c|c|}
\hline Vehicle Category & \% of Pedestrian Accidents \\
\hline \hline Motorcycle & 0.28 \\
\hline Agricultural Vehicle & 0.09 \\
\hline Construction Vehicle & 0.09 \\
\hline Small Passenger Car & 73.36 \\
\hline Minibus & 5.74 \\
\hline Bus & 0.65 \\
\hline Truck & 7.77 \\
\hline Dual Purpose Vehicle & 12.02 \\
\hline Total & 100.00 \\
\hline
\end{tabular}

According to vehicle license type. Private vehicles were involved in $61.4 \%$ of total pedestrian accidents while public vehicles were involved in $32.9 \%$.

According to driver license type. Drivers with private license type were involved in $45.6 \%$ of pedestrian accidents while drivers with public license type or higher were 
involved in $50.3 \%$ of pedestrian accidents. It should be noted that unlicensed drivers were involved in $4.1 \%$ of pedestrian accidents showing that more enforcement is needed for this category in the city.

According to speed limit. The majority of pedestrian accidents have occurred at low speed limits of $40 \mathrm{~km} / \mathrm{hr}$ $(65.2 \%)$ and $50 \mathrm{~km} / \mathrm{hr}(27.8 \%)$ as shown in Table 6. However, drivers tend to show more noncompliance at lower speed limits. Koushki [18] reported that high speed caused $17 \%$ of pedestrian accidents, and speed was cited as the major cause for pedestrian fatalities in Kuwait. Knowles et al. [20] found that around $90 \%$ of fatal pedestrian collisions on London's roads during the period 2006-2010 have occurred on roads with speed limits of $48 \mathrm{~km} /$ hour or lower. Jensen [21] found that the proportion of pedestrians killed in crashes increases in step with the speed limit.

Table 6. Pedestrian Accidents According to Speed Limit

\begin{tabular}{|c|c|}
\hline Speed Limit & \% of Pedestrian Accidents \\
\hline \hline 30 & 3.15 \\
\hline 40 & 65.16 \\
\hline 50 & 27.76 \\
\hline 60 & 2.95 \\
\hline 70 & 0.59 \\
\hline 80 & 0.39 \\
\hline$\geq 90$ & 0.00 \\
\hline Total & 100.00 \\
\hline
\end{tabular}

According to pedestrian actions. The majority of pedestrians $(59 \%)$ were hit by vehicles while crossing the road as shown in Table 7. Without clear and marked crosswalks, which is the case at most Irbid streets, pedestrians cross the streets randomly resulting in more pedestrian accidents.

According to driver faults. The major identified driver fault was "not giving priority to pedestrians" with $37 \%$ followed by "incorrect reversing" with $4.6 \%$, and then "exceeding speeding limit" with $2.3 \%$ as shown in Table 8.

According to driver gender. Male drivers accounted for $97.5 \%$ of pedestrian accidents. This was expected since female drivers constitute a small proportion of Jordanian drivers.

According to driver age. A high proportion of involved drivers in pedestrian accidents refer to young drivers (18-30 years old) with about $48 \%$ as shown in Table 9.

\section{CONCLUSIONS}

Based on the results of the analysis for pedestrian accidents in Irbid City - Jordan, it can be concluded that:

- Children less than 15 years old, accounted for the highest pedestrian accident rate as compared to other age groups.
Table 7. Pedestrian Accidents According to Pedestrian Actions

\begin{tabular}{|l|c|}
\hline \multicolumn{1}{|c|}{ Pedestrian Action } & \% of Pedestrian Accidents \\
\hline \hline Crossing the Road & 59.00 \\
\hline Crossing In front of a Parked Vehicle & 4.66 \\
\hline Working on the Road & 0.81 \\
\hline Playing on the Road & 3.05 \\
\hline Pushing or Towing a Carriage & 0.32 \\
\hline Walking on the Road & 5.14 \\
\hline Crossing at A Zebra Crossing & 0.17 \\
\hline Getting in or out of a Vehicle & 1.45 \\
\hline Walking on Sidewalks & 0.97 \\
\hline Riding a Bicycle & 2.73 \\
\hline Off Road & 2.09 \\
\hline Walking inside a Bus terminal & 6.27 \\
\hline Crossing from an Incorrect Location & 1.93 \\
\hline Others & 11.41 \\
\hline & 100.00 \\
\hline
\end{tabular}

Table 8. Pedestrian Accidents According to Driver Faults

\begin{tabular}{|l|c|}
\hline \multicolumn{1}{|c|}{ Drivers' Faults } & $\begin{array}{c}\text { \%o of Pedestrian } \\
\text { Accidents }\end{array}$ \\
\hline \hline Not Giving Priority to Pedestrians & 37.02 \\
\hline Exceeding Speed Limit & 2.30 \\
\hline Using Incorrect Lane & 2.29 \\
\hline Incorrect Bending and Turning & 0.95 \\
\hline Driving Under the Influence of Alcohol/Drugs & 0.19 \\
\hline Failing to Comply with Obligatory Traffic Signs & 0.57 \\
\hline Not Giving Priority to Vehicles & 0.76 \\
\hline Incorrect Reversing & 4.58 \\
\hline Others & 51.34 \\
\hline \multicolumn{1}{|c|}{ Total } & 100.00 \\
\hline
\end{tabular}

- The majority of pedestrian accidents occurred at midblock locations, during clear and sunny weather, on dry surface, and during daylight conditions.

- $\quad$ Most of pedestrian accidents have resulted into slight and medium injuries.

- Males accounted for higher number of pedestrian accidents as compared to females.

- $\quad$ Pedestrian accidents were more frequent during the time period 12:00-3:00 PM, on Thursdays, and during July. 
Table 9. Pedestrian Accident Causalities According to Driver Age Groups

\begin{tabular}{|c|c|}
\hline Age Group & \% of Pedestrian Accident Causalities \\
\hline \hline$<18$ & 0.74 \\
\hline $18-20$ & 2.50 \\
\hline $20-25$ & 20.87 \\
\hline $25-30$ & 22.63 \\
\hline $30-35$ & 15.58 \\
\hline $35-40$ & 12.43 \\
\hline $40-45$ & 7.98 \\
\hline $45-50$ & 6.96 \\
\hline $50-55$ & 4.08 \\
\hline $55-60$ & 3.06 \\
\hline $60-65$ & 1.77 \\
\hline$>65$ & 1.40 \\
\hline Total & 100.00 \\
\hline
\end{tabular}

- $\quad$ Small passenger cars and dual-purpose vehicles were involved in the majority of pedestrian accidents.

- $\quad$ Private cars accounted for higher number of pedestrian accidents as compared to public cars.

- Drivers with private license type were involved in the majority of pedestrian accidents.

- $\quad$ The majority of pedestrian accidents have occurred at low speed limits of 40 and $50 \mathrm{~km} / \mathrm{hr}$.

- The majority of pedestrians were hit by vehicles while crossing the road and the driver fault "not giving priority to pedestrians" was responsible for the highest percentage of pedestrian accidents.

- $\quad$ Male drivers accounted for higher number of pedestrian accidents as compared to female drivers.

- $\quad$ The majority of involved drivers in pedestrian accidents were young drivers (18-30 years old).

\section{CONFLICT OF INTEREST}

The authors confirm that this article content has no conflicts of interest.

\section{ACKNOWLEDGEMENTS}

The authors are very grateful to the Deanship of Research at the Jordan University of Science \& Technology for funding this research and to Irbid Municipality and Traffic Police for providing the data.

\section{REFERENCES}

[1] JTI, Traffic Accidents in Jordan 2010, Jordan Traffic Institute, Amman, Jordan, June 2011.
[2] D. Shinkle, "Transportation Review: Bicycle and Pedestrian Safety", National Conference of State Legislatures Report, Washington DC, USA, 2012.

[3] J. Ibrahim, H. Day, J. Hirshon, and M. El-Setouhy, "Road riskperception and pedestrian injuries among students at Ain Shams University, Cairo, Egypt", J. Inj. Violence Res., vol. 4, no. 2, pp. 65-72, July 2012.

[4] NHTSA, "Traffic Safety Facts 2010 Data: Pedestrians", Report Number: DOT-HS-811-625, National Highway Traffic Safety Administration, Washington DC, USA, 2012.

[5] European Transport Safety Council, "Unprotected Road Users: a Key Concern of Road Safety", Road Safety Performance Index (Flash 19) Report, ARRB Group Limited, Vermont South, Victoria, 2011.

[6] Choueiri, R. Lamm, G. Choueiri, and B. Choueiri, "Pedestrian accidents: a 15-year survey from the United States and Western Europe", ITE Inst. Transp. Eng., J., vol. 63, no. 7, pp. 36-42, July 1993.

[7] Chandran, T. Sousa, Y. Guo, D. Bishai, and F. Pechansky, "Road traffic deaths in Brazil: rising trends in pedestrian and motorcycle occupant deaths", Traffic Inj. Prev., vol. 13, no. sup1, pp. 11-16, 2012.

[8] C.V. Zegeer, and M. Bushell, "Pedestrian crash trends and potential countermeasures from around the world", Accid. Anal. Prev., vol. 44, no. 1, pp. 3-11, January 2012.

[9] V. Wickramasinghe, A. Priyankara, and S. Dissanayake, Evaluation of pedestrians' illegal road-crossing behavior in developing countries using conjoint analysis, Transportation Research Board 91st Annual Meeting, 2012.

[10] LaPlante, R. Van Houten, S. Meekins, T. Gustafson, and D. Cleveland, "Sharing the road: optimizing pedestrian and bicycle safety and vehicle mobility", Report Number RC-1572, Michigan Dept. of Transportation, Lansing, Michigan, 2012.

[11] R. Viola, M. Roe, and H. Shin, "New York City pedestrian safety study \& action plan", Report Number 657387134, New York Dept. of Transportation, New York, 2010.

[12] Truong, T. Long, and S. Somenahalli, "Using GIS to identify pedestrian-vehicle crash hot spots and unsafe bus stops", J. Public Trans., vol. 14, no. 1, pp. 99-114, January 2011.

[13] Nieuwesteeg, and A. McIntyre, "Exploring the pedestrian crash problem from the perspective of injured pedestrians", Australasian Road Safety Research Policing Education Conference, 2010.

[14] E. Obaidat, "Analysis of pedestrian accidents in Irbid City", M.S. thesis, Jordan University of Science and Technology, Irbid, Jordan, 2003.

[15] K. Jadaan, and A. Nicholson, "Relationships between road accidents and traffic flows in an urban network", Traffic Eng. Control., vol. 33, no. 9, pp. 507-511, September 1992.

[16] H. Al-Masaeid, "Characteristics and Costs of Road Accidents" in: Jordan, Safety on Roads: International Conference (SORIC' 98), 1998, pp. 306-310.

[17] S. Al-Senan, G. Ergun, and A. Al-Khabbaz, "Characteristics of pedestrian accidents in selected cities of Saudi Arabia", Transp. Res. Rec., no. 1405, pp. 49-55, 1993.

[18] P. Koushki, O. Al-Saleh, S. Yaseen, and M. Ali, On Fatal and Injurious Pedestrian Accidents, Transportation Research Board $80^{\text {th }}$ Annual Meeting, 2001.

[19] Z. Al-Momani, "Evaluation of pedestrian related traffic control measures in Greater Amman", M.S. thesis, Jordan University of Science and Technology, Irbid, Jordan,1998.

[20] L. Knowles, R. Cuerden, and E. Delmonte, "Analysis of police collision files for pedestrian fatalities in London (2006-2010)", Report Number PPR620, Transport Research Laboratory, London, UK, 2012.

[21] S. Jensen, "Pedestrian Safety in Denmark", Transportation Research Board $79^{\text {th }}$ Annual Meeting, 1999. 